\section{(6) OPEN ACCESS}

PAPER

\title{
Elective ventilation for organ donation: law, policy and public ethics
}

\author{
John Coggon
}

\begin{abstract}
Correspondence to
Dr John Coggon,

Southampton Law School,

University of Southampton, Southampton, SO17 1BJ, UK; john.coggon@soton.ac.uk
\end{abstract}

Received 31 July 2012 Revised 4 November 2012 Accepted 9 November 2012 Published Online First 7 December 2012

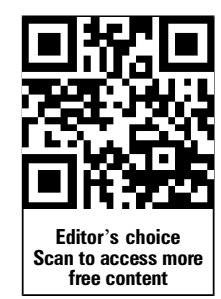

To cite: Coggon J. J Med Ethics 2013;39:130-134.

\begin{abstract}
This paper examines questions concerning elective ventilation, contextualised within English law and policy. It presents the general debate with reference both to the Exeter Protocol on elective ventilation, and the considerable developments in legal principle since the time that that protocol was declared to be unlawful. I distinguish different aspects of what might be labelled elective ventilation policies under the following four headings: 'basic elective ventilation'; 'epistemically complex elective ventilation'; 'practically complex elective ventilation'; and 'epistemically and practically complex elective ventilation'. I give a legal analysis of each. In concluding remarks on their potential practical viability, I emphasise the importance not just of ascertaining the legal and ethical acceptability of these and other forms of elective ventilation, but also of assessing their professional and political acceptability. This importance relates both to the successful implementation of the individual practices, and to guarding against possible harmful effects in the wider efforts to increase the rates of posthumous organ donation.
\end{abstract}

\section{INTRODUCTION}

As a strategy for increasing transplantation rates in England, practices that might fall under the label 'elective ventilation' have been tainted by the notoriety of the Exeter Protocol, described by TG Feest and colleagues in a paper published in The Lancet in $1990 .^{1}$ David Price summarises procedures under that protocol as follows:

[Elective ventilation] protocols target patients in deep irreversible coma and believed to be dying imminently of intracranial haemorrhage. Such patients are transferred, with the consent of relatives, to intensive care units so that artificial ventilation can be initiated as soon as respiratory arrest occurs, thus preserving the organs until brain death can be established. ${ }^{2}$

Strong concerns have been raised about this strategy. Although the chances of it causing harm are low, one of the risks is of putting the patient into a persistent vegetative state (PVS), which is such a serious harm that there are obvious fears that the risk may not be worth running. ${ }^{3}$ Furthermore, judged by legal standards at the time, the Exeter Protocol was plainly unlawful; family members had no power to consent under English law, and the best interests standard as judges then conceived it could not permit the practice. ${ }^{2}$ And while the Protocol's advocates made clear ethical arguments, these were posited in a utilitarian framework; rather than consider substantive benefit to the patient, the justification was based on there being no harm to the patient and significant overall benefit (ie, through benefit to the organ recipients, and thus a wider social benefit). ${ }^{2}$ While some personhood theories may de-problematise deontological critiques of such an ethic, ${ }^{4}$ it is doubtful that this would receive overwhelming social or professional endorsement, given (among other things) that the patient would be legally alive, and the tragic situation in which decisions would need to be made. (In other words, I would suggest that it is unimaginable that a clinician would feel able to justify the adoption of such utilitarian reasoning by asserting, for example, that the patient is not a 'Harrisian person'.)

However, debates about elective ventilation in the UK have reawakened. ${ }^{5}$ The surrounding law has changed substantially. ${ }^{6}$ Furthermore, considerable effort has been given to changing social and professional attitudes to organ donation. ${ }^{3}$ 7-9 Against that backdrop of changing views and changed legal principle, there is a significant danger that 'received wisdoms' concerning the Exeter Protocol will foreclose proper consideration of the viability of elective ventilation strategies. In this paper, I therefore consider the law as it relates to practices that may be labelled, or associated with, elective ventilation for potential posthumous organ donors. I outline four scenarios and discuss the law as it would apply to them. I then discuss the conclusions with a view to policy and public ethics.

\section{ELECTIVE VENTILATION AS A TRANSPLANTATION POLICY}

The term 'elective ventilation' may be understood in various ways. In public and academic debates, it is most widely taken to denote the initiation of invasive measures to ventilate an irreversibly unconscious patient in order to optimise the chances of successful posthumous organ donation, following a decision that continued treatment can provide no benefit to the patient's own health or clinical prospects. It is common to find plain assertions in academic papers and public documents stating that elective ventilation is outright unlawful.

Things are not, however, so straightforward. For a start, it should be noted that elective ventilation is not itself a term of legal art in English law; the law does not in terms 'say' anything about a practice of this name. Furthermore, any exploration of the legality of potential policies now must account for the significant changes in principle since the mid-1990s. We should treat with caution arguments made at that time concerning the unlawfulness of 
the Exeter Protocol. The law governing treatment of adult patients who lack decision-making capacity continues to apply a best interests test (save in instances where an advance directive applies or if there is a formally appointed proxy decision-maker, in accordance with the Mental Capacity Act 2005). However, as explained below, best interests now enjoys a much richer and more nuanced understanding than it did in the $1990 \mathrm{~s},{ }^{10}$ at which point it was largely reducible to so-called 'medical interests'. Acknowledging the very broad scope of best interests in English law it is hard for anyone to claim with certainty that elective ventilation as described at the start of this section would always and in every case be unlawful.

Given the developments in principle, and also the span of practical discussions on organ policy and elective ventilation, I do not limit the scope of this paper just to the institution of ventilation. While some ethical and professional perspectives differ on this, at law there is no automatic distinction to be drawn between a practice of continuing ventilation and starting ventilation; in either case, a decision simply has to be made in accordance with a patient's best interests. So if the law does debar a particular instance of elective ventilation, this is not due to a universal principle on instituting ventilation in and of itself; exposure to a heightened risk of harm in initiation rather than continuation may make the difference, but this would in principle be a question assessed on a case by case basis, rather than as an abstract and general matter. It is more instructive, therefore, to widen the net, even if some readers do not consider what follows to be solely about what they would term elective ventilation. Probably the most contested medical-ethical questions in what follows concern instituting, as opposed to continuing, invasive measures, and handling uncertainty concerning the substantive content of a patient's best interests. These matters receive attention, but to cover all of the relevant ground it is most useful to enumerate four situations, all of which repay specific legal and ethical analysis in the current debate. My aim in describing them as I do is to facilitate analysis by separating some of the different ethical concerns that arise. They all apply to patients who lack decision-making capacity, and who have no specific advance-directive or decision-making proxy appointed to consent on their behalves. Thus, there can be no role for consent. With each potential practice, there is, at least on the face of things, increasing ethical complexity. I label them respectively: 'basic elective ventilation', 'epistemically complex elective ventilation', 'practically complex elective ventilation', and 'epistemically and practically complex elective ventilation':

Basic elective ventilation: Here we have a dying, critically ill patient in the intensive care unit (ICU). It is decided that continued, life-sustaining ventilation is no longer clinically beneficial to the patient. However, it is recognised that the patient would be a suitable organ donor after he dies, and it is known that this decision is commensurate with what the patient would have wanted to happen. A further decision is therefore made to continue ventilation, which causes no harm to the patient even as it confers no therapeutic benefit. During this continuation, a surgical retrieval team has time to make necessary preparations to perform a transplant. The modest intervention of continued ventilation, withdrawn slightly later with a result of a slightly delayed cardiac death, followed by better co-ordinated transplantation, optimises the chances of a successful posthumous donation.

Epistemically complex elective ventilation: Here the process differs from basic elective ventilation because it is not known whether the dying patient wanted to become a posthumous donor. Given such uncertainty, the patient's life is prolonged following the judgment of therapeutic futility not because it is inferred that he would want that in order to optimise chances of successful donation. Rather, it is prolonged so that his (probable) wishes can be established, in case it transpires that still further prolongation of ventilation is indicated in order to optimise the chances of successful posthumous donation. Thus, an intervention that is judged to be clinically futile is continued because it is thought to be in the best interests of the patient to assure that more facts about his best interests and the question of posthumous donation are established before he is allowed to die.

Practically complex elective ventilation: Here the process differs from basic elective ventilation because the dying, critically ill patient is not in ICU. It is expected that the withdrawal of treatment from the patient will cause cardiac death, and clinically this is the indicated course of action. Although continued treatment is considered to be clinically futile, however, it is known that the patient would want to become a posthumous organ donor. He is thus moved to ICU where his condition is stabilised, and withdrawal-and consequent cardiac death-occur after the transplantation team is prepared.

Epistemically and practically complex elective ventilation: Here the process combines complicating elements of the previous two. It is not known whether the dying, critically ill patient, who is not in ICU, would wish to become a posthumous donor. Following the decision that continued treatment confers no therapeutic benefit, the patient is moved to ICU and is ventilated first to allow time to make an informed best interests assessment of whether further continued stabilisation and ventilation for posthumous transplantation is indicated, and second to provide such continuation should that finding be affirmative.

\section{LEGAL ANALYSIS}

Although decisions about elective ventilation are made with a view to posthumous donation, the decision-making necessarily relates to would-be donors before death; the patient at the centre of the decision may be dying, but he is legally alive. Accordingly, decisions must be made on the basis of his best interests as this standard is formulated at law. Given the focus of our discussion, it is important to stress again that analysis must be made according to contemporary legal standards. While the legal literature and policy statements on organ donation span decades, principle now is markedly distinct to that even 15 years ago, and thus only limited reliance can be placed on conclusions expressed in older arguments. This point presents itself most starkly if we contrast the findings in two papers by David Price, the first published in $1996,{ }^{2}$ the second in $2011 .^{6}$ The practical conclusions of each are importantly different. Price's 2011 paper provides the most notable contemporary statement and analysis of end-of-life law as it relates to organ donation, and its contrast with his 1996 argument demonstrates clearly the substantial changes in principle, and their implications for practice and policy developments now.

\section{The development of best interests at law}

Price's 1996 paper in part responds to the Exeter Protocol, and more generally suggests agreement with a view that all of the elective ventilation procedures described above are unlawful. Best interests at that time, especially given dicta in the famous case of Bland, ${ }^{11}$ suffered only a very narrow interpretation, which would now be described simply as covering patients' 'medical interests'. The ethical reasoning used to support the Exeter Protocol was utilitarian, and could not be reconciled with the law's treatment of living people as individuated rights-holders who could only be interfered with in such circumstances if there was a freely given consent or a narrowly 
construed 'clinical benefit' supporting their best interests, even where no harm would be done to them. A best interests judgment could not allow elective ventilation, altruism could not be imputed, and there was no scope for proxy or implied consent to govern the situation. ${ }^{2}$

Since Bland, however, the principle concerning best interests as understood in English law has undergone an impressive evolution. While a detailed history is not possible in this paper, it bears stressing that as judges have come to interpret the idea of best interests, an increased emphasis can be seen to have fallen on two sorts of 'de-medicalisation'. First, the courts will not defer unquestioningly to medics' appraisals of best interests, even where these are supported by other physicians. In English medical lawyers' terms, best interests cannot be reduced to a 'Bolamised' question of clinical judgment. Second, the courts have been clear that they will not allow best interests to be reduced just to 'medical' or 'clinical' interests. Instead, a more 'holistic' appraisal is required, that looks to a much richer concept of welfare. This finds clarity particularly in statements made by the courts around the turn of the century, saying, for example, that best interests must incorporate broader ethical, social, moral and welfare considerations. ${ }^{12}$ Of course this change does not allow patients who lack capacity to become simple means to others' ends, but it does allow for non-therapeutic benefits, including non-physical and nonexperiential benefits, to feature in decision-making. Perhaps the most notable common law decision in this regard is the case of Ahsan. ${ }^{13}$ Here the High Court found that a Muslim patient in PVS should be cared for at home rather than in hospital. The patient was completely unconscious, and thus would not be aware of the move. Nor would there be any therapeutic advantage in it. Nevertheless, the court found that she would benefit as care at home was commensurate with her religious faith; it was the course indicated by the particular values that she held when she had had capacity. Since Absan, the common law understanding of best interests has assumed statutory footing with the coming into force of the Mental Capacity Act 2005. Of particular note, section 4(6) of the Act demands that decision-makers, when assessing best interests, account for the patient's past and present wishes, and the beliefs and values that would influence his decision if he were making it. It is therefore very hard to say, in the abstract, that a given measure such as those described in this paper would never be in any patient's best interests as judged at law.

\section{Best interests and basic elective ventilation}

Applied to what I am here calling basic elective ventilation, current legal requirements do not simply suggest its permissibility, but that it can be mandatory. The justification is personcentred, not utilitarian. ${ }^{6}$ It relies on respect for finding and applying the patient's own values. As such, the potential legality of basic elective ventilation is not in doubt. There will be clear instances where it is the indicated course of action and medical decision-makers will thus be bound, according to the patient's best interests, to continue harmless, modest measures to optimise the chances of successful donation after treatment is withdrawn. ${ }^{14-16}$ In 1996 it would have rightly been judged unlawful to support such a policy simply on the basis of reasoning such as that advanced in defence of the Exeter Protocol. Furthermore, English law's best interests standard at that time was not apt to include altruistic values. ${ }^{2}$ At the time of writing (2012), however, basic elective ventilation's lawfulness is clear when it does no harm and advances the patient's own interests in achieving an end that he valued. ${ }^{6}$
Best interests and epistemically complex elective ventilation Epistemically complex elective ventilation also falls within the bounds of being lawful. The General Medical Council's guidance on end-of-life care says at paragraph 31, under the heading 'Addressing uncertainty':

If there is a reasonable degree of uncertainty about whether a particular treatment will provide overall benefit for a patient who lacks capacity to make the decision, the treatment should be started in order to allow a clearer assessment to be made. ${ }^{17}$

It stands to reason that as far as the patient is concerned, it could be fundamentally important that consideration go to organ donation. Necessarily this is a probabilistic question. However, people are not merely benefited by substantial outcomes, but also by being exposed to probable benefits or defended from probable harms, whether or not these finally eventuate. For the question under issue, there are clear arguments that would support epistemically complex elective ventilation. There is a good enough chance in cases of meaningful uncertainty that a patient would want measures instituted to enhance posthumous donation, that a best interests appraisal would indicate continued ventilation while the inquiry was made.

\section{Best interests and practically complex elective ventilation} Peter Watkinson and colleagues describe how:

Patients who present with untreatable or rapidly lethal conditions are currently not admitted to, or treated on, ICUs because such management prolongs dying and may result in harm. Common practice has been to facilitate organ donation by prolonging life sustaining treatment only in those already in the ICU who have expressed a wish to donate their organs. ${ }^{5}$

Their paper is written in response to recent guidance from the UK's National Institute for Health and Clinical Excellence. ${ }^{8}$ This guidance, which healthcare professionals must take into account, but which does not substitute their obligation to make appropriate decisions given a particular patient's specific circumstances, advocates for what I label practically complex elective ventilation (as well as doing so for epistemically complex elective ventilation). In other words, it suggests an extension from the common practice described in the quoted text.

Watkinson et al question the lawfulness of this practice where it is performed with a view simply to optimising the chances of successful posthumous donation. They cite the contention surrounding the Exeter Protocol, and the Department of Health's claims in the 1990s that the practice was unlawful. As we have seen, such legal advice should not now be taken as conclusive, given the radical differences in legal principle concerning best interests between then and now. Movement of the patient to ICU of itself is not something that can now be said to be ruled out by law.

In their discussion of the law, Watkinson et al also refer to the report of a consensus meeting, which was published in $2010 .^{18}$ They say this report 'reaffirmed' the Department of Health's 1995 guidance, condemning the Exeter Protocol as unlawful. It should be stressed, however, that the consensus meeting's legal opinion here does not entirely 'reaffirm' the 1995 view: it relates not to movement of the patient, which it recognises could be lawful, but to the question of whether intubation and ventilation of patients could be commenced (as opposed to continued) following a clinical futility decision regarding life-sustaining treatment. This gives rise to separate, legally relevant concerns regarding exposure to the risk of harm, which may debar a best 
interests judgment that would favour measures to enhance the chances of posthumous donation. However, this additional concern does not give rise to an intrinsic legal problem with the proposal of moving a patient to ICU. It should be added that while it may be argued that instituting ventilation in these circumstances would always be unlawful, the law in this area cannot be said to make such a proposition obviously true.

\section{Best interests and epistemically and practically complex elective ventilation}

Finally, we have the question of the lawfulness of moving patients to ICU when it is unknown whether or not their best interests would be served by measures to increase the chances of successful posthumous donation. In accordance with the above, it should be clear that while this seems to be more complex, legally it is possible for such a course of action to be in a patient's best interests.

It is important to stress, however, that questions beyond lawfulness are crucial here. For analytical purposes, I have separated the above practices, but there are two fundamental issues that need to be addressed before these arguments can have further effect. First, as I have emphasised, the best interests standard is person-centred, and its imperatives derive from considerations particular to the patient in question. As such, it is possible to make general legal observations, and to suggest default courses of action, but the proper process in any given situation will necessarily be subject to individual judgment. That a practice may in principle be potentially lawful does not mean it will be in a given situation. Second, norms and permissible practices in this area are not static. It is necessary to consider legal argument, but developments in policy need to account for wider questions of public ethics, with a view to what is practically possible, and to avoiding negative outcomes. I shall give brief mention to this last point as I draw together my conclusions.

\section{PUBLIC ETHICS, POLICY AND PRACTICES: BEYOND THE LAW}

If the desired-and realisable-numbers of organ transplantations are to be achieved, a diversity of continued developments in practice is needed. ${ }^{3}$ Policy-makers need to consider what may (and may not) be done to increase transplants both from living and dead donors. Full deliberation includes tackling questions of what is ethically viable, practically possible, politically realistic, and professionally and socially acceptable. Consideration must be given to legal frameworks, healthcare infrastructures, and healthcare practices. Beyond the widespread academic debates, there have in recent years been a range of high-profile public reports and guidance documents on these matters in the UK. ${ }^{3-9}$ Although there are some marked differences in these reports, in substance there seems to be more agreement than disagreement. Optimism can be drawn from a wide public-ethical and political accord on many issues. Perhaps most notably, there seems to be a broad consensus on the following: that financial incentives to living donors should not be permitted; that the right 'philosophy' underpinning posthumous donation is that it should be the norm; and that nevertheless the system is one of respectful pluralism that permits those with objections against donation not to have their organs 'harvested' against their will. Although there are philosophical disagreements about the wisdom and coherence of each of these propositions, from a policy perspective a broad consensus that will lead to better outcomes is clearly preferable to stubborn and sub-optimal stasis given dogged intellectual disagreement that results in avoidable death and suffering. ${ }^{19}$
Building on this consensus, continued efforts are needed in many areas of strategy and policy-development. Readers of journals such as this are all too familiar with the idea that knowing what the law 'says' does not speak to what is ethically permissible or mandatory (familiar to the extent that sometimes the law can be too quickly ignored when it provides at the very least an important fact about the world that requires consideration in a practical, action-guiding ethical argument!). Equally, it is widely recognised that there are all too many questions in healthcare law to which the rather unhelpful answer is 'The law on this is not entirely clear.'; or 'It depends...'.

A further, related point that demands acknowledgment is that policies and practices are only sensibly implemented if we allow for more than legality and morality. There is increasing recognition in contemporary bioethical debates of the importance of public ethics. ${ }^{20-25}$ This can be understood not simply as moral agents having to be able to account for their morality and its implications; to give reasons. Far beyond an exercise in pure morality, it is a political study, examining the force, limits, and implications of different sorts of normative and other argument in the practical situations we find (including in healthcare). ${ }^{26}$

In this paper, I have explained how English law is able to accommodate various practices that fall within the ambit of 'elective ventilation'. I have not here made the moral arguments, but would simply assert (and in another place have to defend the position) that the moral case can also be made in its favour. Of significance in my concluding comments, however, is that legal and moral legitimacy are not all that we need before we can accept the unrolling of a policy. The debates on public ethics remind us how important it is to look to the acceptability of new policies, both to professionals who will be required to work under them, and to the wider public. Organ donation policy is controversial, and leads to heated argument and potentially deep suspicion both of the healthcare profession and of the State. Before implementing a policy in this area, it is essential that due attention be given to its acceptability (its actual, not its 'in principle', acceptability) and probable effects. Past experience-for example concerning the Exeter Protocol, or the organ retention scandals ${ }^{27}{ }^{28}$ - tells us that politically these different policies and strategies are not entirely separable. If it is to become practice, it is crucial to know that an elective ventilation policy would be lawful. But it is fundamental also to assure it will not lead to any political or professional backlash. This is not just because of defending the policy itself, but also to protect wider advances in the overall progress to an ethically better, more respectful, and less wasteful organ donation system.

Acknowledgements Thanks to Muireann Quigley and Dominic Wilkinson for comments on an earlier draft, to the Wellcome Strategic Programme in the Human Body, Its Scope, Limits and Future (grant number WT087439/Z/08/Z), held in the Institute for Science, Ethics, and Innovation, at the University of Manchester, for its support during the writing of this paper, and to the Yale and Hastings Visiting Scholars Program.

Funding None.

Competing interests None.

Provenance and peer review Not commissioned; externally peer reviewed.

Open Access This is an Open Access article distributed in accordance with the terms of the Creative Commons Attribution (CC BY 3.0) license, which permits others to distribute, remix, adapt and build upon this work, for commercial use, provided the original work is properly cited. See: http://creativecommons.org/ licenses/by/3.0/

\section{REFERENCES}

1 Feest TG, Riad HN, Collins $\mathrm{CH}$, et al. Protocol for increasing organ donation after cerebrovascular deaths in a district general hospital. Lancet 1990;335:1133-5. 
2 Price D. Contemporary transplantation initiatives: where's the harm in them? J Law Med Ethics 1996;24:139-49.

3 British Medical Association Medical Ethics Committee. Building on progress: where next for organ donation policy in the UK? London: BMA, 2012.

4 Harris J. The value of life. London: Routledge and Kegan Paul, 1985.

5 Watkinson P, McKechnie S, Wilkinson D, et al. Actively delaying death to increase organ donation. BMJ 2012;344:1179.

6 Price D. End-of-Life treatment of potential organ donors: paradigm shifts in intensive and emergency care. Med Law Rev 2011;19:86-116.

7 Organ Donation Taskforce. Organs for transplants: a report from the organ donation taskforce. London: Department of Health, 2008.

8 National Institute for Health and Clinical Excellence. Organ donation for transplantation: Improving donor identification and consent rates for deceased organ donation. London: NICE, 2011.

9 Nuffield Council on Bioethics. Human bodies: donation for medicine and research. London: Nuffield Council on Bioethics, 2011

10 Coggon J. Best interests, public interest, and the power of the medical profession. Health Care Anal 2008;16:219-32.

11 Airedale NHS Trust v. Bland [1993] 1 All ER 821.

12 In re S (Adult Patient: Sterilisation) [2001] Fam 15, per Dame Elizabeth Butler-Sloss $P$, at 28.

13 Ahsan v. University Hospitals Leicester NHS Trust [2007] PIQR P19.

14 Coggon J, Brazier M, Murphy $P$, et al. Best interests and potential organ donors. BMJ 2008;336:1346-7.

15 Coggon J, Murphy P. Ante-Mortem issues affecting deceased donation: an ethico-legal perspective. In: Farrell A, Price D, Quigley M, eds. Organ shortage: principles, pragmatism and practice. Cambridge: Cambridge University Press, 2011:136-48.
16 Coggon J. Doing what's best: organ donation and intensive care. In: Danbury C, Waldmann C, Newdick C, Lawson A, eds. Ethics and law in critical care. Oxford: Oxford University Press, 2010:213-31.

17 General Medical Council. Treatment and care towards the end of life: good practice in decision making. London: GMC, 2010.

18 Intensive Care Society and British Transplantation Society. Donation after circulatory death. 2010. http://www.ics.ac.uk/intensive_care_professional/standards_and_ guidelines/dcd (accessed 20 July 2012).

19 Harris J, Brazier M. Does ethical controversy cost lives? In: Farrell A, Price D, Quigley $M$, eds. Organ shortage: principles, pragmatism and practice. Cambridge: Cambridge University Press, 2011:15-33.

20 Coggon J. Assisted dying and the context of debate: 'Medical Law' versus 'End-of-Life Law'. Med Law Rev 2010;18:541-63.

21 Montgomery J. Reflections on the nature of 'public ethics'. Camb Q Healthc Ethics (forthcoming 2013).

22 Wolff J. Ethics and public policy: a philosophical inquiry. London: Routledge, 2011.

23 Tannery CA. Bioethics in the public square: reflections on the how. J Med Ethics 2012;38:439-41.

24 Harris J. Life in the cloud and freedom of speech. J Med Ethics (forthcoming)

25 Farsides B. Negotiating change: organ donation in the United Kingdom. In: Farrell A, Price D, Quigley M, eds. Organ shortage: principles, pragmatism and practice. Cambridge: Cambridge University Press, 2011:215-26.

26 Coggon J. What makes health public? A critical evaluation of moral, legal, and political claims in public health. Cambridge: Cambridge University Press, 2012.

27 Bristol Inquiry Interim Report, Removal and Retention of Human Material. 2000. http://www.bristol-inquiry.org.uk (accessed 20 July 2012).

28 Report of the Inquiry into the Royal Liverpool Children's Hospital (Alder Hey). 2001. http://www.rclinquiry.org.uk (accessed 20 July 2012). 